\title{
Sinai-Ruelle-Bowen measures for contracting Lorenz maps and flows
}

\author{
by \\ Roger J. METZGER ${ }^{1}$ \\ Instituto de Matemática y Ciencias Afines - IMCA, Jr. Ancash 536, \\ Casa de las Trece Monedas, Lima 1, Peru \\ Manuscript received 11 March 1999
}

ABSTRACT. - We consider a large class of one-dimensional maps arising from the contracting Lorenz attractors for three dimensional flows: the eigenvalues $\lambda_{2}<\lambda_{1}<0<\lambda_{3}$ of the flow at the singularity satisfy $\lambda_{1}+\lambda_{3}<0$ (instead of $\lambda_{1}+\lambda_{3}>0$ as in the classical geometric Lorenz models). Such flows were studied by A. Rovella who showed that non-uniform expansiveness is a persistent form of behavior (positive Lebesgue measure sets of parameters). Using mainly expansiveness, we prove the existence of absolutely continuous measures invariant under these maps, and from this fact we are able to construct Sinai-RuelleBowen measures for the original flows that generate them.

(C) 2000 L'Association Publications de l'Institut Henri Poincaré. Published by Elsevier B.V. All rights reserved

RÉSUMÉ. - Nous considérons une classe importante de transformations uni-dimensionelles provenant d'attracteurs de Lorenz contractants des flots en dimension 3 : les valeurs propres $\lambda_{2}<\lambda_{1}<0<\lambda_{3}$ du flot au point singulier satisfont $\lambda_{1}+\lambda_{3}<0$ (au lieu de $\lambda_{1}+\lambda_{3}>0$, comme dans les modèles geòmétriques de Lorenz standards). Ces flots ont été etudiés par A. Rovella qui a montré que l'expansion non-uniforme a un comportament persistant (ensembles de paramètres de mesure positive). En utilisant cette expansion non-uniform, nous démonstrons l'existence

\footnotetext{
${ }^{1}$ E-mail: metzger@uni.edu.pe.
} 
de mesures invariantes par ces transformations qui sont absolutement continues. De ce fait, nous déduisons l'existence de mesures SRB pour le flots qui les induisent.

(C) 2000 L'Association Publications de l'Institut Henri Poincaré. Published by Elsevier B.V. All rights reserved

\section{INTRODUCTION}

Sinai-Ruelle-Bowen measures, SRB or physical measures, are those measures for what the Birkhoff averages converge to a constant for a large Lebesgue set. More precisely: if $f: M \rightarrow M$ is a transformation on a manifold $M$, we call an $f$-invariant measure $\mu$ an SRB measure if there exists a positive Lebesgue measure set $B(\mu)$ of points $x \in M$ such that

$$
\lim _{n \rightarrow \infty} \frac{1}{n} \sum_{i=1}^{n} \varphi\left(f^{i}(x)\right)=\int_{M} \varphi \mathrm{d} \mu \quad \text { for every } \varphi \in C_{0}(M, \mathbb{R}) .
$$

and the set $B(\mu)$ is called (ergodic) basin of attraction of $\mu$.

For a flow $f^{t}: M \rightarrow M$ the definition is

$$
\lim _{T \rightarrow \infty} \frac{1}{T} \int_{0}^{T} \varphi\left(f^{t}(x)\right) \mathrm{d} t=\int_{M} \varphi \mathrm{d} \mu \quad \text { for every } \varphi \in C_{0}(M, \mathbb{R}) .
$$

Lorenz flows are related to the system studied in [8], as a truncation of a Navier-Stokes equation. Guckenheimer and Williams [3] introduced a geometric model called expanding Lorenz attractor, in which they suppose that the eingenvalues $\lambda_{2}<\lambda_{1}<0<\lambda_{3}$ at the singularity of the flow satisfy the expanding condition $\lambda_{1}+\lambda_{3}>0$. In [11], the expanding conditions is replaced by the contracting one $\lambda_{1}+\lambda_{3}<0$. The general assumptions used to construct the geometric models, also permit the reduction of the 3-dimensional problem, first to a 2-dimensional Poincare section and then to a one-dimensional map. These maps are also called Lorenz-like.

We will prove the existence of a unique and ergodic absolutely continuous invariant measure (a.c.i.m.) for certain one-dimensional Lorenz-like maps (Theorem A). After this, we will relate these results to the case of flows and construct an SRB measure in this case too. Since the a.c.i.m. found for the one-dimensional case is unique, the SRB measure constructed for the flow is also unique. 
We will use four properties of the one-dimensional Lorenz-like maps studied by [11]. More precisely. Let $I \subset[-1,1]$ be a compact interval and $f: I \rightarrow I$ be a map such that $f(I) \subset I$ with a discontinuity at the origin. Set $c_{k}^{ \pm}=\lim _{x \rightarrow 0^{ \pm}} f^{k}(x)$ for $k \geqslant 0$. So, we will require $f$ to satisfy conditions (A0)-(A3) below.

(A0) Outside the origen $f$ is of class $C^{3}$ and with negative Schwarzian derivative, and also satisfies

$$
K_{2}|x|^{s-1} \leqslant f^{\prime}(x) \leqslant K_{1}|x|^{s-1}
$$

for some constants $K_{1}, K_{2}$ and $s$ with $s>1$.

(A1) $\left(f^{n}\right)^{\prime}\left(c_{1}^{ \pm}\right)>\lambda_{c}^{n}$, for some $\lambda_{c}>1$, and for $n \geqslant 1$.

(A2) $\left|f^{n-1}\left(c_{1}^{ \pm}\right)\right|>\mathrm{e}^{-\alpha n}$ some $\alpha$ small enough, and all $n \geqslant 1$.

(A3) For any interval $J \subset I$ there exists a number $n(J)>0$ such that $I_{*} \subset f^{n}(J)$ ( $f$ is topologically mixing on $\left.I_{*}=\left[c_{1}^{+}, c_{1}^{-}\right]\right)$.

Rovella in [11] showed the existence of a one parameter family of maps which exhibit conditions (A0)-(A2) in a set of parameters of positive Lebesgue measure. For a slightly smaller class of maps it is also true that conditions (A1) and (A2) implies condition (A3). This fact is proved in Lemma A. We work here with such a continuous family of maps, but the arguments, and then the conclusions, remains valid for a larger class of maps with negative Schwarzian derivative and with a finite number of non degenerate critical points.

It is clear from our definitions that if $\mu$ is an absolutely continuous invariant measure for $f$ and ergodic then it is an SRB measure. Now, we can state our main theorem.

THEOREM 1.1 (Theorem A). - Under conditions (A0)-(A3), $f$ admits an absolutely continuous invariant probability measure. This measure is unique and ergodic.

The basic strategy is to reduce the non-uniform hyperbolicity of the dynamics of our maps to that of piecewise uniformly expanding maps. That is what conditions (A1)-(A2) are for, which express a kind of expansiveness. Condition (A3) is used principally for the uniqueness. The techniques used here resemble that of Viana [14]. Frequently, we will refer to this work for proofs that do not need major modifications.

The main difference in our aproach comes from the fact that our map is not continuous and also has two critical orbits. We overcome the problem defining the tower to keep track of both orbits, resulting in a tower extension with two blocks. It is also possible to work with maps that 
have more discontinuities or singularities if they have properties similar to (A0)-(A3).

SRB measures were first proved to exist for Anosov systems [13] and then for general uniformly hyperbolic diffeomorphisms [12] and flows [1]. For these systems there are finitely many SRB measures $\mu_{1}, \ldots, \mu_{N}$, and their basin of attractions cover Lebesgue almost all the phase space $M$. Moreover, they are stochastically stable (see Kifer [5,6]). The same is true for the expanding Lorenz attractor as proved in Chapter 4 of [6]. We shall show that the contracting Lorenz atrractor is also stochastically stable in a forthcoming work. Here is to be pointed aut that J. Palis conjectured that every dynamical system can be approximated by another having only finitely many attractors, supporting physical measures that describe the time average of Lebesgue almost all points, and that the statistical properties of this measures are stable under small random perturbations, see $[9,15]$. In that sense our present and next works can be seen as a contribution to, or at least as an example of, Palis conjecture.

Theorem A is proved in Sections 2 through 5. In Section 6 we will establish some results on decay of correlations. This is made to complete the description of the dynamics of the one dimensional map $f$. In the last section, we will conclude relating this result to the contracting Lorenz attractor.

\section{SETTINGS}

For our constructions and proofs we need several constants, let us fix them here. First, suppose that the constant $\alpha$ in (A2) has been taken small enough so that $\mathrm{e}^{s \alpha /(s-1)}<\lambda_{c}^{1 / s}$. In order to construct the tower extension, we fix $\beta \in((s+1) \alpha / s, s \alpha /(s-1))$, and $\lambda>1$. Up to here these constants are enough for the definitions, but we will need other constants to establish the expanding behavior of our tower extension. Let $\rho>\mathrm{e}^{\alpha}$ such that $\mathrm{e}^{-\alpha} \lambda_{c}^{1 / s}>\lambda \rho$, and also let $1<\sigma \leqslant \sigma_{0}$ and $0<\delta \leqslant \delta_{0}$, where $\sigma_{0} \in(1, \lambda)$ and $\delta_{0}$ is much less than $\alpha$. These constants are given by Lemma 3.1 later on this section.

Our next step is the definition of the tower extension (cf. [14]). The main feature of the tower is that it transforms our map $f$, which is not uniformly expansive, to a map $\hat{f}$ that is uniformly expansive. For this, set $B_{0}=I$ and $B_{k}^{ \pm}=\left[c_{k}^{ \pm}-\mathrm{e}^{-\beta k}, c_{k}^{ \pm}+\mathrm{e}^{-\beta k}\right]$ for each $k \geqslant 1$. We let $E_{k}^{ \pm}=B_{k}^{ \pm} \times\{k\}$ and set $\hat{I}=\left(\bigcup_{k \geqslant 1} E_{k}^{+}\right) \cup\left(\bigcup_{k \geqslant 1} E_{k}^{-}\right) \cup E_{0}$. Note that the critical point 0 is not contained in $B_{k}^{ \pm}$for any $k \geqslant 1$, since (A2) implies $\left|c_{k}^{ \pm}\right|>\mathrm{e}^{-\alpha k}>\mathrm{e}^{-\beta k}$. 
We want to define $\hat{f}: \hat{I} \rightarrow \hat{I}$ to be a tower extension in the sense of [14]. But, since our initial map has a discontinuity, we should establish that a point $(x, 0)$ which is ready to 'climb a level' should go up to level $E_{1}^{+}$if $x>0$, and to level $E_{1}^{-}$if $x<0$.

The precise expression for $\hat{f}(x, k)$ is the following:

$$
\hat{f}(x, k)= \begin{cases}(f(x), 0) & \text { if }|x| \geqslant \delta \text { and } k=0, \\ (f(x), 0) & \text { if } f(x) \text { is not in } B_{k+1}^{+} \text {for }(x, k) \in E_{k}^{+} \\ & \text {or if } f(x) \text { is not in } B_{k+1}^{-} \text {for }(x, k) \in E_{k}^{-}, \\ (f(x), k+1) & \text { otherwise, } \\ & \text { and with the additionally conditions that, } \\ & (f(x), k+1) \in E_{k+1}^{+} \text {if }(x, k) \in E_{k}^{+} \text {or } \\ & (f(x), k+1) \in E_{k+1}^{-} \text {if }(x, k) \in E_{k}^{-} .\end{cases}
$$

Typically, a point $(x, 0)$ moves around in the ground level $E_{0}$ for a while until it hits $(0, \delta) \times\{0\}$ or $(-\delta, 0) \times\{0\}$ at some time $m \geqslant 0$. Then it starts climbing the tower in the following way

$$
\hat{f}^{m+j}(x, 0)=\left(f^{m+j}(x), j\right), \quad 0 \leqslant j \leqslant n,
$$

where $\left(f^{m+j}(x), j\right) \in E_{j}^{+}$if $f^{m}(x)<0$ and $\left(f^{m+j}(x), j\right) \in E_{j}^{-}$if $f^{m}(x)>0$.

Unless $f^{m}(x)$ coincides with the critical point 0 , the integer $n$ is finite and in the next iterate the orbit falls back to the ground level, that is, $\hat{f}^{m+n+1}(x, 0)=\left(f^{m+n+1}(x), 0\right)$. Observe that we must have $n \geqslant H(\delta)$ for some integer $H(\delta) \geqslant 1$ which can be made arbitrarily large by choosing $\delta$ small enough.

Now we define the cocycle $\omega_{0}$. First, we set $\omega_{0}(x, 0)=1$ for every $x \in B_{0}$. Given any point $(x, k) \in E_{k}^{ \pm}, k \geqslant 1$, there are two possibilities:

(1) There exists $z$ with $|z|<\delta$ such that $\hat{f}^{k}(z, 0)=(x, k)$, in which case we define

$$
\omega_{0}(x, k)=\lambda^{k}\left(\left(f^{k}\right)^{\prime}(z)\right)^{-1} .
$$

It's easy to see that if $z$ exist then it is unique, and has the additional property that $z<0$ if $(x, k) \in E_{k}^{+}$and $z>0$ if $(x, k) \in E_{k}^{-}$.

(2) There is no such $z$, in which case we simply set $\omega_{0}(x, k)=0$.

For each $k \geqslant 1$ we shall denote $W_{k}^{ \pm}=\left\{x \in B_{k}^{ \pm}: \omega_{0}(x, k)>0\right\}$ and $W_{0}=\left\{x \in B_{0}: \omega_{0}(x, 0)>0\right\}$ (i.e., $W_{0}=B_{0}$ ). 
Note that every $W_{k}^{ \pm}$is an interval whose closure contains $c_{k}^{ \pm}$. We also write

$$
\widehat{W}=\left(\bigcup_{k \geqslant 1} W_{k}^{+} \times\{k\}\right) \cup\left(\bigcup_{k \geqslant 1} W_{k}^{-} \times\{k\}\right) \cup W_{0} .
$$

Now, we associate to $\omega_{0}$ the Borel measure $m_{0}=\omega_{0} m$ where $m$ is the Lebesgue measure on $\hat{I}$. Moreover if we denote $|\cdot|$ the metric in $\hat{I}$ induced by the standard metric in $I$, we can associate to $\omega_{0}$ the Riemannian metric $\|\cdot\|_{(x, k)}=\omega_{0}(x, k)|\cdot|$.

It results from the definition that $\omega_{0}$ and $m_{0}$ are both supported on the subset $\widehat{W}$. Reflecting the fact that points in $\hat{I} \backslash \widehat{W}$ are transient for $\hat{f}$, and play no role as far as asymptotic behavior is concerned. Let us note that certain points in the ground level are also transient, specifically, $\hat{f}(\widehat{W})$ does not intersect $\left\{\left(f(-\delta), c_{1}^{-}\right) \cup\left(c_{1}^{+}, f(\delta)\right)\right\} \times\{0\}$. In order to see this, if there exist $(x, k) \in \widehat{W}$ such that

$$
\hat{f}(x, k) \in\left\{\left(f(-\delta), c_{1}^{-}\right) \cup\left(c_{1}^{+}, f(\delta)\right)\right\} \times\{0\},
$$

then $f(x) \in\left(f(-\delta), c_{1}^{-}\right) \cup\left(c_{1}^{+}, f(\delta)\right)$, and in that case we must have $x \in(-\delta, \delta)$ if $\delta>0$ is small enough so that $c_{2}^{-}<f(-\delta)<c_{1}^{-}$and $c_{1}^{+}<$ $f(\delta)<c_{2}^{+}$. In order to have $\hat{f}(x, k) \in E_{0}$ we must have $k+1>H(\delta)$. Assume that

$$
0<\delta<\left(2\left(16 K_{1}\right)^{1 /(s-1)}\right)^{-1}
$$

so that

$$
\text { length }\left(B_{k}\right) \leqslant 2 \mathrm{e}^{-\beta k} \leqslant\left(2\left(16 K_{1}\right)^{1 /(s-1)}\right)^{-1}
$$

then, since $x \in B_{k}^{ \pm} \cap(-\delta, \delta)$, the interval $B_{k}$ must be contained in $\left(-\left(16 K_{1}\right)^{-1 /(s-1)},\left(16 K_{1}\right)^{-1 /(s-1)}\right) \backslash\{0\}$, and we have

$$
f^{\prime}(y) \leqslant K_{2}|y|^{s-1} \leqslant K_{1}\left(\frac{1}{\left(16 K_{1}\right)^{1 /(s-1)}}\right)^{s-1} \leqslant 1 / 16,
$$

so $f^{\prime}(y) \leqslant 1 / 16$ for every $y \in B_{k}^{ \pm}$.

On the other hand, from the fact that $\mathrm{e}^{\beta}<\mathrm{e}^{s /(s-1)}<\lambda_{c}^{1 / s}<2$ we have

$$
\left|f(x)-c_{k+1}^{ \pm}\right| \leqslant \frac{1}{16} \mathrm{e}^{-\beta k} \leqslant \frac{\mathrm{e}^{\beta}}{16} \mathrm{e}^{-\beta(k+1)} \leqslant \frac{1}{8} \mathrm{e}^{-\beta(k+1)},
$$

which means that $\hat{f}(x, k) \in E_{k+1}^{ \pm}$contradicting the choice of $(x, k)$. 
Now, for any point $(y, l)$ such that $\hat{f}(y, l) \in \widehat{W}$, we set

$$
g(y, l)=\frac{1}{f^{\prime}(y)} \frac{\omega_{0}(y, l)}{\omega_{0}(\hat{f}(y, l))} .
$$

Clearly, $g(y, l)>0$ with this definition. Moreover, when $(y, l) \in \widehat{W}$, $1 / g(y, l)$ is the Jacobian of $\hat{f}$ at $(y, l)$, with respect to the metric $\|\cdot\|$ (or equivalently, with respect to the measure $\left.m_{0}\right)$.

Now, given a measurable function $\varphi: \hat{I} \rightarrow \mathbb{R}$ we define

$$
\begin{aligned}
\operatorname{var}(\varphi) & =\sum_{k \geqslant 1} \operatorname{var}\left(\left.\varphi\right|_{E_{k}^{+}}\right)+\sum_{k \geqslant 1} \operatorname{var}\left(\left.\varphi\right|_{E_{k}^{-}}\right)+\operatorname{var}\left(\left.\varphi\right|_{E_{0}}\right), \\
\sup (\varphi) & =\sup _{k \geqslant 1}\left(\sup \left(\left.\varphi\right|_{E_{k}^{ \pm}}\right)\right), \quad \text { and } \\
\int \varphi \mathrm{d} m_{0} & =\sum_{k \geqslant 1} \int_{E_{k}^{+}} \varphi \omega_{0} \mathrm{~d} m+\sum_{k \geqslant 1} \int_{E_{k}^{-}} \varphi \omega_{0} \mathrm{~d} m+\int_{E_{0}} \varphi \omega_{0} \mathrm{~d} m .
\end{aligned}
$$

Now we define the $B V$-norm of $\varphi$ as

$$
\|\varphi\|_{B V}=\operatorname{var}(\varphi)+\sup (|\varphi|)+\int|\varphi| \mathrm{d} m_{0},
$$

and let $\widehat{B V}=\left\{\varphi: \hat{I} \rightarrow \mathbb{R}:\|\varphi\|_{B V}<\infty\right\}$. With this definition, it is clear that $\widehat{B V}$ is a Banach space.

Finally, we describe the transfer operator $\mathcal{L}_{0}$ associated to $\hat{f}$. Given $\varphi \in \widehat{B V}$ and $(x, k) \in \widehat{W}$, we set

$$
\mathcal{L}_{0} \varphi(x, k)=\sum_{\hat{f}(y, l)=(x, k)} \frac{\varphi(y, l) \omega_{0}(y, l)}{f^{\prime}(y) \omega_{0}(x, k)}=\sum_{\hat{f}(y, l)=(x, k)} g \varphi(y, l) .
$$

Observe that for $k=0$ there may be infinitely many terms. Then we extend $\mathcal{L}_{0} \varphi$ to $\hat{I} \backslash \widehat{W}$ by asking that it be constant on each connected component of $B_{k}^{ \pm} \backslash W_{k}^{ \pm}$for each $k \geqslant 1$.

More precisely, let $a_{k}<b_{k}$ be the endpoints of the interval $W_{k}^{ \pm}$, then we define

$$
\mathcal{L}_{0}(\varphi)(x, k)= \begin{cases}\limsup _{y \rightarrow a_{k}^{+}} \mathcal{L}_{0} \varphi(y, k), & \text { if } y \leqslant a_{k} \\ \liminf _{y \rightarrow b_{k}^{-}} \mathcal{L}_{0} \varphi(y, k), & \text { if } y \geqslant b_{k}\end{cases}
$$

This definition is made so that $\operatorname{var}\left(\mathcal{L}_{0} \varphi\right)$ and $\sup \left|\mathcal{L}_{0} \varphi\right|$ are not affected if we restrict ourselves to $\widehat{W}$. The variation of $\mathcal{L}_{0} \varphi$ over $E_{k}^{ \pm}$coincides 
with the variation of $\mathcal{L}_{0} \varphi$ over $W_{k}^{ \pm}$and a similar fact is true for the supremum of $\mathcal{L}_{0} \varphi$. Of course the same holds for $\int \mathcal{L}_{0} \varphi \mathrm{d} m_{0}$ because $m_{0}$ is supported on $\widehat{W}$. In particular, the duality relation

$$
\int \mathcal{L}_{0}(\varphi) \psi \mathrm{d} m_{0}=\int \varphi \psi \circ \hat{f} \mathrm{~d} m_{0}
$$

whenever the integrals make sense, is not affected by this convention.

Clearly, $\mathcal{L}_{0}$ is a nonnegative operator, in the sense that it maps nonnegative functions to nonnegative functions. So, relation (1) also implies that $\mathcal{L}_{0}$ is not increasing with respect to the $L^{1}$-norm, that is

$$
\int \mathcal{L}_{0}(\psi) \mathrm{d} m_{0} \leqslant \int \mathcal{L}_{0}(|\psi|) \mathrm{d} m_{0}=\int|\psi| \mathrm{d} m_{0} \quad \text { for every } \psi \in \widehat{B V} .
$$

\section{EXPANSION LEMMAS}

In this section we state two key lemmas on the expanding behavior of certain iterates of the map $f$. They are formulate in the same form as [14], because they are also true for the maps we are considering here.

LEMMA 3.1 (Vi 5.2). - There are constants $\sigma_{0}>1, b>0$ and $\delta_{0}>0$ such that for any $0<\delta \leqslant \delta_{0}$ there is $c(\delta)>0$ such that, given any $x \in I$ and $n \geqslant 1$

(1) if $x, f(x), \ldots, f^{n-1}(x) \notin(-\delta, \delta)$ then $\left(f^{n}\right)^{\prime}(x) \geqslant c(\delta) \sigma_{0}^{n}$;

(2) if in addition, $f^{n}(x) \in(-\delta, \delta)$ then $\left(f^{n}\right)^{\prime}(x) \geqslant b \sigma_{0}^{n}$.

Proof. - It was proved in other form by A. Rovella in [11], see Lemma $1,1.1,1.2$ and their proofs, in the mentioned article.

Now, we take the constant $\delta$ in the definition of the tower, satisfying $0<\delta \leqslant \delta_{0}$, and fix $\sigma \in\left(1, \delta_{0}\right]$, and we have

LEMMA 3.2 (Vi 5.3). - There is a constant $C>0$ such that for any $z \in(-\delta, 0)$ and $k \geqslant 1$

(i) if $\left|f^{j}(z)-c_{j}^{-}\right| \leqslant \mathrm{e}^{-\beta j}$ for every $1 \leqslant j \leqslant k$ then

$$
\frac{1}{C} \leqslant \frac{\left(f^{k}\right)^{\prime}(f(z))}{\left(f^{k}\right)^{\prime}\left(c_{1}^{-}\right)} \leqslant C
$$

(ii) if in addition, $\left|f^{k+1}(z)-c_{k+1}^{-}\right| \geqslant \mathrm{e}^{-\beta(k+1)}$, then

$$
\left(f^{k}\right)^{\prime}(f(z)) \geqslant \frac{1}{C} \lambda_{c}^{k} \quad \text { and } \quad\left(f^{k+1}\right)^{\prime}(z) \geqslant \frac{1}{C} \rho^{k} \lambda^{k} M
$$


where $M=\mathrm{e}^{-\alpha}\left(s /\left(C K_{1}\right)^{(s-1) / s} \cdot K_{2}\right.$.

And similar results hold if $z \in(0, \delta)$ and $\left|f^{j}(z)-c_{j}^{+}\right| \leqslant \mathrm{e}^{-\beta j}$.

Proof. - Let us proof part (i). First of all note that

$$
\frac{\left(f^{k}\right)^{\prime}(f(z))}{\left(f^{k}\right)^{\prime}\left(c_{1}^{-}\right)}=\prod_{j=1}^{k} \frac{f^{\prime}\left(f^{j}(z)\right)}{f^{\prime}\left(c_{j}^{-}\right)}=\prod_{j=1}^{k}\left(1+\frac{f^{\prime}\left(f^{j}(z)\right)-f^{\prime}\left(c_{j}^{-}\right)}{f^{\prime}\left(c_{j}^{-}\right)}\right)
$$

so we only have to get a uniform bound for

$$
\sum_{j=1}^{k}\left|\frac{f^{\prime}\left(f^{j}(z)\right)-f^{\prime}\left(c_{j}^{-}\right)}{f^{\prime}\left(c_{j}^{-}\right)}\right|
$$

Now, $f$ has negative Schwarzian derivative in $B_{j}^{-}$since $0 \notin B_{j}^{-}=$ $\left[c_{j}^{-}-\mathrm{e}^{\beta j}, c_{j}^{-}+\mathrm{e}^{\beta j}\right]$, and as long as $f^{j}(z) \in B_{j}^{-}$we have that

$$
\begin{aligned}
\left|\frac{f^{\prime}\left(f^{j}(z)\right)-f^{\prime}\left(c_{j}^{-}\right)}{f^{\prime}\left(c_{j}^{-}\right)}\right| & \leqslant\left|f^{\prime \prime}(y)\right|\left|\frac{f^{j}(z)-c_{j}^{-}}{f^{\prime}\left(c_{j}^{-}\right)}\right| \\
& \leqslant A|y|^{s-2}\left|\frac{f^{j}(z)-c_{j}^{-}}{f^{\prime}\left(c_{j}^{-}\right)}\right| .
\end{aligned}
$$

Then from condition (A0) we obtain:

$$
\sum_{j=1}^{k}\left|\frac{f^{\prime}\left(f^{j}(z)\right)-f^{\prime}\left(c_{j}^{-}\right)}{f^{\prime}\left(c_{j}^{-}\right)}\right| \leqslant \frac{A}{K_{2}} \sum_{j=1}^{k} \frac{\mathrm{e}^{-\beta j}}{\mathrm{e}^{-\alpha j}} .
$$

The right side is bounded provided that $\beta>\alpha$ (remember we have chosen $(s /(s-1)) \alpha>\beta>((s+1) / s) \alpha$, so $\beta>\alpha)$. This proves part (i).

Now, to prove part (ii), first observe that the first claim in (ii) is a direct consequence of part (i) and (A1). The second one can be obtained as follows. Let $z$ and $k$ be as in the statement, then

$$
\begin{aligned}
\left(f^{k+1}\right)^{\prime}(z) & =\left(f^{k}\right)^{\prime}(f(z)) \cdot f^{\prime}(z) \geqslant K_{2}|z|^{s-1}\left(f^{k}\right)^{\prime}(f(z)) \\
& \geqslant \frac{K_{2}}{C}|z|^{s-1}\left(f^{k}\right)^{\prime}\left(c_{1}^{-}\right) .
\end{aligned}
$$

We can estimate the value of $|z|$ from the inequality

$$
\begin{aligned}
\mathrm{e}^{-\beta(k+1)} & \leqslant\left|f^{k+1}(z)-c_{k+1}^{-}\right|=\left(f^{k}\right)^{\prime}(\xi)\left|f(z)-c_{1}^{-}\right| \\
& \leqslant K_{1} C\left(f^{k}\right)^{\prime}\left(c_{1}^{-}\right) \frac{|z|^{s}}{s}
\end{aligned}
$$


for some $\xi \in\left(f(z), c_{1}^{-}\right)$from the Mean Value Theorem. For this $\xi$ there exists $y$ satisfying the conditions in part (i) and such that $f(y)=\xi$. The last inequality is due to (A0). So the inequality above is a consequence of the Mean Value Theorem and part (i).

Rewriting the equation, it stands that:

$$
|z|^{s} \geqslant \frac{s}{C K_{1}}\left|\left(f^{k}\right)^{\prime}\left(c_{1}^{-}\right)\right|^{-1} \mathrm{e}^{-\beta(k+1)} .
$$

Combining this last inequality with (2) we obtain

$$
\left(f^{k+1}\right)^{\prime}(z) \geqslant \frac{K_{2}}{C}\left(\frac{\mathrm{e}^{-\beta} s}{C K_{1}}\right)^{(s-1) / s}\left(\lambda_{c}^{1 / s}\right)^{k} \cdot \mathrm{e}^{-\beta k((s-1) / s)} .
$$

Since $\beta<(s /(s-1)) \alpha$ and $\lambda_{c}^{1 / s} \cdot \mathrm{e}^{-\alpha}>\lambda \rho$ we have

$$
\begin{aligned}
\left(f^{k+1}\right)^{\prime}(z) & \geqslant \frac{K_{2}}{C}\left(\frac{\mathrm{e}^{-\beta} s}{C K_{1}}\right)^{(s-1) / s}\left(\lambda_{c}^{1 / s} \mathrm{e}^{-\alpha}\right)^{k} \\
& \geqslant \frac{K_{2}}{C} \mathrm{e}^{-\alpha}\left(\frac{s}{C K_{1}}\right)^{(s-1) / s} \lambda^{k} \rho^{k},
\end{aligned}
$$

leading to

$$
\left(f^{k+1}\right)^{\prime}(z) \geqslant \frac{1}{C} \rho^{k} \lambda^{k} M,
$$

where $M=\mathrm{e}^{-\alpha}\left(s /\left(C K_{1}\right)\right)^{(s-1) / s} \cdot K_{2}$.

This end the proof of Lemma 3.2.

We denote by $\mathcal{P}^{(n)}$ the partition of $\hat{I}$ into monotonicity intervals of $\hat{f}^{n}$, for $n \geqslant 1$, and characterized in the following way: For every $k \geqslant 1$, set

$$
U_{k}^{ \pm}=\left\{(x, k) \in E_{k}^{ \pm}: \hat{f}(x, k)=(f(x), k+1)\right\} .
$$

Let $D_{k}^{L \pm}, D_{k}^{R \pm}$ be the two connected components of $E_{k}^{ \pm} \backslash U_{k}^{ \pm}$, that is, points in $U_{k}^{ \pm}$are sent by $\hat{f}$ to an upper level of the tower, whereas points in $D_{k}^{L \pm} \cup D_{k}^{R \pm}$ are mapped down to the ground level $E_{0}$. For $k=0$ we set

$$
\begin{array}{ll}
U_{0}^{L}=(-\delta, 0] \times\{0\}, & U_{0}^{R}=(0, \delta] \times\{0\}, \\
D_{0}^{L}=[q,-\delta] \times\{0\}, & D_{0}^{R}=[\delta,-q] \times\{0\} .
\end{array}
$$

Then we set

$$
\mathcal{P}^{(1)}=\left\{U_{k}^{ \pm}, D_{k}^{L \pm}, D_{k}^{R \pm}, k \geqslant 1\right\} \cup\left\{U_{0}^{L}, U_{0}^{R}, D_{0}^{L}, D_{0}^{R}\right\} .
$$


Now, for any $n>1$, we set $\mathcal{P}^{(n)}$ to be the $n$th iterate by $\hat{f}$ of $\mathcal{P}^{(1)}$, that is,

$$
\mathcal{P}^{(n)}\left(\xi_{1}\right)=\mathcal{P}^{(n)}\left(\xi_{2}\right) \quad \text { if } \mathcal{P}^{(1)}\left(\hat{f}^{i}\left(\xi_{1}\right)\right)=\mathcal{P}^{(1)}\left(\hat{f}^{i}\left(\xi_{2}\right)\right)
$$

for each $0 \leqslant i<n$.

From now on, we will always assume that every $\eta \in \mathcal{P}^{(n)}$ has positive length. Moreover, the intersection of $\eta$ with $\widehat{W}$ is either empty or an interval with positive length. Note that in order to have this it suffices that the $\hat{f}$-orbits of points

$$
\left(0^{ \pm}, 0\right), \quad( \pm \delta, 0), \quad\left(c_{k}^{ \pm}+\mathrm{e}^{-\beta k}\right) \quad \text { and } \quad\left(c_{k}^{ \pm}-\mathrm{e}^{-\beta k}\right) \quad \text { for } k \geqslant 1
$$

be two-by-two disjoint injective sequences on $\hat{I}$, which can always be obtained by slightly modifying $\beta$ and $\alpha$ if necessary (so as to avoid a countable set of relations involving these two constants).

It follows from our definitions that if $(x, k) \in U_{k}^{ \pm} \cap \widehat{W}, k \geqslant 1$, and $z \in(-\delta, \delta) \backslash\{0\}$ such that $\hat{f}^{k}(z, 0)=(x, k)$, then

$$
\begin{aligned}
g(x, k) & =\frac{1}{f^{\prime}(x)} \frac{\omega_{0}(x, k)}{\omega_{0}(\hat{f}(x, k))}=\frac{1}{f^{\prime}(x)} \frac{\lambda^{k}\left|\left(f^{k}\right)^{\prime}(z)\right|^{-1}}{\lambda^{k+1}\left|\left(f^{k+1}\right)^{\prime}(z)\right|^{-1}} \\
& =\frac{1}{\lambda} \frac{\left|\left(f^{k+1}\right)^{\prime}(z)\right|}{\left(f^{k}\right)^{\prime}(z) f^{\prime}\left(f^{k}(z)\right)}=\frac{1}{\lambda} .
\end{aligned}
$$

The same is true if $(x, 0) \in(-\delta, \delta)=U_{0}^{L} \cup U_{0}^{R}$.

On the other hand, if $(x, k)$ is in $D_{k} \cap \widehat{W}, k \geqslant 1$, and $z$ as before $\left(D_{k}\right.$ here means some of the $D_{k}^{ \pm L}$ or $D_{k}^{ \pm R}$ ) then

$$
\begin{aligned}
g(x, k) & =\frac{1}{f^{\prime}(x)} \frac{\omega_{0}(x, k)}{\omega_{0}(\hat{f}(x, k)}=\frac{\lambda^{k}\left|\left(f^{k}\right)^{\prime}(z)\right|^{-1}}{1} \\
& =\frac{\lambda^{k}}{f^{\prime}\left(f^{k}(z)\right)\left(f^{k}\right)^{\prime}(z)}=\frac{\lambda^{k}}{\left(f^{k+1}\right)^{\prime}(z)} \leqslant \frac{C}{M} \rho^{-k} .
\end{aligned}
$$

The last inequality is consequence of Lemma 3.2.

Observe that $k \geqslant H(\delta)$, where $H(\delta)$ is the minimum height from which orbits in $(-\delta, \delta) \times\{0\}$ can fall down to $E_{0}$ (see Section 5.3 in [14]). We suppose that $\delta$ is small, so $H(\delta)$ is large and implies $(C / M) \rho^{-k}<$ $1 / \lambda<1$. Therefore, $g(x, k)<1 / \lambda<1$ in all the situations above, which express the uniformly expanding character of $\hat{f}$, because $1 / g$ acts as the Jacobian of $\hat{f}$ respect to $m_{0}$. 
We shall also need the iterated version $g^{n}$ of $g$, which is defined by

$$
g^{(n)}(\xi)=g(\xi) g(\hat{f}(\xi)) \cdots g\left(\hat{f}^{n-1}(\xi)\right)=\frac{1}{\left(f^{n}\right)^{\prime}} \frac{\omega_{0}(\xi)}{\omega_{0}(\hat{f}(\xi))}
$$

for every $\xi=(x, k)$ such that $\hat{f}^{i}(\xi) \in \widehat{W}$ for $1 \leqslant i \leqslant n$.

The following three lemmas will be stated without proofs because they are similar to the corresponding lemmas in [14].

LEMMA 3.3 (Vi 5.4).-

(1) Let $\gamma \subset \eta \in \mathcal{P}^{(n)}$ be such that $\hat{f}(\gamma) \subset E_{0}$ for every $0 \leqslant j \leqslant n$. Then

$$
\sup _{\gamma} g^{(n)} \leqslant \begin{cases}C \sigma^{-n}, & \text { if } \hat{f}^{n}(\gamma) \subset(-\delta, \delta) \times\{0\}, \\ C(\delta) \sigma^{-n}, & \text { in general. }\end{cases}
$$

Moreover, $\operatorname{var}_{\gamma} g^{(n)} \leqslant 2 \sup _{\gamma} g^{(n)}$.

(2) Let $\gamma \subset \eta \subset \widehat{W}$ for some $\eta \in \mathcal{P}^{(n)}$ and let $0 \leqslant l \leqslant \min \{k, n-1\}$ be such that $\hat{f}(\gamma) \subset E_{k-l+i}^{ \pm}$for $0 \leqslant i \leqslant l$ and $\hat{f}(\gamma) \subset E_{0}$ for $l<i \leqslant n$. Then

$$
\sup _{\gamma} g^{(n)} \leqslant \begin{cases}C \lambda^{-l} \rho^{-k} \sigma^{-n+l+1}, & \text { if } \hat{f}^{n}(\gamma) \subset(-\delta, \delta) \times\{0\}, \\ C(\delta) \lambda^{-l} \rho^{-k} \sigma^{-n+l+1}, & \text { in general. }\end{cases}
$$

Moreover, $\operatorname{var}_{\gamma} g^{(n)} \leqslant 2 \sup _{\gamma} g^{(n)}$.

(3) Let $\gamma \subset \eta \cap \widehat{W}$ for some $\eta \in \mathcal{P}^{(n)}$ and let $l \geqslant 0$ such that $\hat{f}^{i}(\gamma) \in$ $E_{l+i}$ for $0 \leqslant i \leqslant n$ then $g^{(n)}=\lambda^{-n}$ on $\gamma$.

LEMMA 3.4 (Vi 5.5). - There is $C>0$ and, for each $n \geqslant 1$, there is $C(n)>0$ such that for every $\varphi \in \widehat{B V}$ and every interval $A \subset E_{0}$,

$$
\begin{aligned}
\operatorname{var}_{A}\left(\mathcal{L}_{0}^{n} \varphi\right) & \leqslant \operatorname{var}\left(\mathcal{X}_{A} \mathcal{L}_{0}^{n} \varphi\right) \\
& \leqslant C \sigma^{-n}(\operatorname{var} \varphi+\sup |\varphi|)+C(n) \int|\varphi| \mathrm{d} m_{0} .
\end{aligned}
$$

LEMMA 3.5 (Vi 5.6). - Given any $\bar{\sigma} \in(1, \sigma)$ there is $C>0$ such that:

$$
\begin{aligned}
& \operatorname{var} \varphi \mathcal{L}_{0}^{n} \varphi \leqslant C \bar{\sigma}^{-n}(\operatorname{var} \varphi+\sup |\varphi|)+C \int|\varphi| \mathrm{d} m_{0}, \\
& \sup \varphi \mathcal{L}_{0}^{n} \varphi \leqslant C \bar{\sigma}^{-n}(\operatorname{var} \varphi+\sup |\varphi|)+C \int|\varphi| \mathrm{d} m_{0},
\end{aligned}
$$

for any function $\varphi \in \widehat{B V}$ and for all $n \geqslant 1$. 


\section{THE MIXING PROPERTY}

We establish (A3) in order to make clear that a mixing property is needed to show uniqueness of the measure and stochastic stability. However, in our present setting, this can be chosen to be a consequence of (A1) and (A2) as explained below.

In [11] it is proved that a one-parameter family of maps $\left\{f_{a}\right\}_{a \in[0,2)}$, satisfying condition (A0) among others, has a positive Lebesgue measure subset $E \subset[0,2)$ such that for all $a \in E, f_{a}$ satisfies (A1) and (A2) with $0 \in E$ as a point of density. This subset can be chosen to satisfy (A3), i.e., the following is true.

LEMMA 4.1 (Lemma A). - In a small enough neighborhood of the density point, iff satisfies (A1) and (A2) then it satisfies (A3).

This makes our construction more relevant since it shows that that conditions (A1)-(A3) are satisfied for a large set of functions, say, for maps in a positive Lebesgue measure set in a one parameter family of maps.

Lemma A seems Lemma 2.1 in [16] so we need properties similar to $\mathrm{P} 1$ and P2 of [16]. Property P1 is the same as Lemma 3.1 and Property $\mathrm{P} 2$ is the contain of Lemma 4.2. To show P2 we need some previous definition that will be use only for the proof of Lemma A.

Let $I_{m}=\left(\mathrm{e}^{-m-1}, \mathrm{e}^{m}\right)$ for $m>0$, let $I_{m}=-I_{-m}$ for $m<0$, and $I_{m}^{+}=I_{m-1} \cup I_{m} \cup I_{m+1}, \delta=\mathrm{e}^{-\Delta}$, with $\Delta \in \mathbb{N}$.

DEFINITION. - Let $p(m)$ be the largest integer $p$ such that

$$
\left|f^{j}(x)-f^{j-1}\left(c_{1}^{+}\right)\right|=\left|f^{j}(x)-c_{j}^{+}\right| \leqslant \mathrm{e}^{-\beta j}, \quad \text { if } m>0,
$$

and

$$
\left|f^{j}(x)-f^{j-1}\left(c_{1}^{-}\right)\right|=\left|f^{j}(x)-c_{j}^{-}\right| \leqslant \mathrm{e}^{-\beta j}, \quad \text { if } m<0,
$$

for $j=1, \ldots, p$ and $x \in I_{m}^{+}$.

The time interval $1, \ldots, p(m)$ is called the bound period for $\mathrm{I}_{m}^{+}$.

LEMMA 4.2. - For each $|m|>\Delta p(m)$ has the following properties.

(a) There is a constant $C_{1}(\alpha, \beta)$ such that:

(i)

$$
\frac{1}{C_{1}} \leqslant \frac{\left(f^{j}\right)^{\prime}(y)}{\left(f^{j}\right)^{\prime}\left(c_{1}^{+}\right)} \leqslant C_{1} \quad \text { if } y \in\left[-1, f\left(\mathrm{e}^{-|m|+1}\right)\right],
$$


(ii)

$$
\frac{1}{C_{1}} \leqslant \frac{\left(f^{j}\right)^{\prime}(y)}{\left(f^{j}\right)^{\prime}\left(c_{1}^{-}\right)} \leqslant C_{1} \quad \text { if } y \in\left[f\left(-\mathrm{e}^{-|m|+1}\right), 1\right]
$$

for $j=0, \ldots, p(m)$.

(b)

$$
\frac{s|m|}{\beta+\log 4}-K \leqslant p(m) \leqslant \frac{s+1}{\beta+\log \lambda_{c}}|m|,
$$

where $K=\left(\beta+\log \left(K_{1} / s\right)+s\right) /(\beta+\log 4)$.

(c)

$$
\left(f^{p+1}\right)^{\prime}(x) \geqslant \exp \left(\left(1-\beta \frac{s+2}{\beta+c}\right)|m|\right)
$$

where $p=p(m)$ and for $x \in I_{m}^{+}$.

Proof. - Suppose $y \in\left[c_{1}^{+}, f\left(\mathrm{e}^{-|m|+1}\right)\right]$ (for $y \in\left[f\left(\mathrm{e}^{-|m|+1}\right), c_{1}^{-}\right]$the proof is similar).

The proofs of parts (a) and (c) are easy consequence of Lemma 3.1. So we only have to prove (b).

For $x \in I_{m}^{+}$we have, assuming $m \geqslant 0$ to fix ideas,

$$
\begin{aligned}
\mathrm{e}^{-\beta p} & \geqslant\left|f^{p}(x)-c_{p}^{+}\right|=\left|f^{p-1}(f(x))-f^{p-1}\left(c_{1}^{+}\right)\right| \\
& =\left(f^{p-1}\right)^{\prime}(y)\left|f(x)-\left(c_{1}^{+}\right)\right|
\end{aligned}
$$

for some $y \in\left[c_{1}^{+}, f(x)\right] \subset\left[-1, f\left(\mathrm{e}^{-|m|+1}\right)\right]$ so,

$$
\begin{aligned}
\left|f^{p}(x)-f^{p-1}\left(c_{1}^{+}\right)\right| & =\left(f^{p-1}\right)^{\prime}(y)\left|f(x)-\left(c_{1}^{+}\right)\right| \geqslant\left(f^{p-1}\right)^{\prime}(y) K_{2} \frac{|x|^{s}}{s} \\
& \geqslant \frac{\left(f^{p-1}\right)^{\prime}\left(c_{1}^{+}\right)}{C_{1}} \frac{K_{2}}{s} \mathrm{e}^{(-|m|-2) s}, \\
\mathrm{e}^{-\beta p} & \geqslant \frac{\lambda_{c}^{(p-1)}}{C_{1}} \frac{K_{2}}{s} \mathrm{e}^{-|m| s} \mathrm{e}^{-2 s} .
\end{aligned}
$$

So we have the following bound for $p$,

$$
\log \left(\frac{K_{2}}{C_{1} s}\right)-|m| s-2 s+\log \lambda_{c} p-\log \lambda_{c} \leqslant-\beta p
$$


that is,

$$
p \leqslant \frac{s|m|}{\log \lambda_{c}+\beta}+\frac{\log \lambda_{c}+2 s-\log \frac{K_{2}}{C_{1} s}}{\log \lambda_{c}+\beta} .
$$

If $|m|$ is large enough we can write,

$$
p \leqslant \frac{(s+1)|m|}{\log \lambda_{c}+\beta}
$$

For the other inequality, from the definition of $p$, there must exists a $z \in I_{m}^{+}$such that

$$
\mathrm{e}^{-\beta(p+1)} \leqslant\left|f^{p}(f(z))-f^{p}\left(c_{1}^{+}\right)\right| \leqslant\left(f^{p}\right)^{\prime}(y)\left|f(z)-\left(c_{1}^{+}\right)\right| .
$$

Supposing that $f^{\prime} \leqslant 4$, we obtain,

$$
\mathrm{e}^{-\beta(p+1)} \leqslant 4^{p} K_{1} \frac{z^{s}}{s} \leqslant 4^{p} \frac{K_{1}}{s} \mathrm{e}^{(-|m|+1) s}
$$

so

$$
-\beta(p+1) \leqslant p \log 4+\log \left(K_{1} / s\right)+-|m|+s
$$

which implies that

$$
p \geqslant \frac{s|m|}{\beta+\log 4}-\frac{\log \left(K_{1} / s\right)+s+\beta}{\beta+\log 4} .
$$

Proof of Lemma A. - In [16], it was used the fact that the fixed point of the map $f$ in $I$ has dense pre-images. We do not have this fixed point for $f$ but we have one for $f^{2}$ (i.e., we have two periodic points of period two). Now, observe that our family of maps can be chose so that we have this fixed point for $f^{2}$ with dense preimages, as required in the arguments of [16]. This is due to the fact that the family (and also the positive Lebesgue measure set satisfying (A1) and (A2)) has as a point of density a map which is conjugated to the transformation $x \rightarrow 2 x \bmod \mathbb{Z}$. So the conclusion remains valid.

\section{ABSOLUTELY CONTINUOUS INVARIANT MEASURES}

Before going into the proofs of our main results, we need the following lemma 
LEMMA 5.1 (Vi 5.7). - The measure $m_{0}=\omega_{0} m$ is a finite measure on $\hat{I}$.

Proof. - It is clear that $m_{0}\left(E_{0}\right)=m\left(E_{0}\right)=m(I)$ is finite. Moreover, for each $k \geqslant 1$

$$
m_{0}\left(E_{k}^{ \pm}\right)=\int_{B_{k}^{ \pm}} \omega_{0}(x, k) \mathrm{d} m(x)=\int_{W_{k}^{ \pm}} \frac{\lambda^{k}}{\left(f^{k}\right)^{\prime}(z)} \mathrm{d} m(x),
$$

where $z \in(-\delta, \delta)$ is uniquely defined by $\hat{f}^{k}(z, 0)=(x, k)$. We change variables $z=f^{k}(x)$, and we get

$$
m_{0}\left(E_{k}^{ \pm}\right)=\int_{Y_{k}^{ \pm}} \lambda^{k} \mathrm{~d} m(z)=\lambda^{k} m\left(Y_{k}^{ \pm}\right),
$$

where $Y_{k}^{+}=\left\{z \in(-\delta, 0): f^{k}(z) \in W_{k}^{+}\right\}$, and $Y_{k}^{-}=\left\{z \in(0, \delta): f^{k}(z) \in\right.$ $\left.W_{k}^{-}\right\}$.

Next, we observe that

$$
\begin{aligned}
2 \mathrm{e}^{-\beta k} & \geqslant m\left(B_{k}^{ \pm}\right) \geqslant m\left(W_{k}^{ \pm}\right) \geqslant \frac{1}{C}\left(f^{k-1}\right)^{\prime}\left(c_{1}^{ \pm}\right) m\left(f\left(Y_{k}^{ \pm}\right)\right) \\
& \geqslant \frac{1}{C} \lambda_{c}^{k-1} \frac{K_{2}}{s} m\left(Y_{k}^{ \pm}\right)^{s},
\end{aligned}
$$

where the third inequality is a consequence of (A0) and (A1). Replacing above, and recalling that we have chosen $\lambda_{c}^{1 / s}>\mathrm{e}^{\alpha} \lambda \rho$ we obtain that

$$
\begin{aligned}
m_{0}\left(E_{k}^{ \pm}\right) & \leqslant\left(2 C \mathrm{e}^{-\beta k} \lambda_{c}^{-k+1} \frac{s}{K_{2}}\right)^{1 / s} \lambda^{k} \leqslant\left(2 C \frac{s}{K_{2}} \lambda_{c}\right)^{1 / s} \mathrm{e}^{-\beta k / s} \rho^{-k} \\
& \leqslant C \rho^{-k}
\end{aligned}
$$

for every $k \geqslant 1$. Since we chose $\rho>1$ the claim follows immediately.

THEOREM 5.1. - The maps $\hat{f}$ and $f$ have absolutely continuous invariant measures $\hat{\mu}_{0}$ and $\mu_{0}$ respectively.

Proof. - The proof of this theorem is contained in [14].

The arguments in [14] assure that $\mathcal{L}_{0}$ has a unique fixed point $\varphi_{0}$ in $\widehat{B V}$. This function is the density of $\hat{\mu}_{0}$ with respect to $m_{0}$. We only make a remark on the fact that we are using the arguments that prove 
the existence of the measures. We are left to prove that $\mu_{0}$ is unique in the space of $f$-invariant probability measures absolutely continuous with respect to Lebesgue. To prove this, we first observe that the measure $\mu_{0}$ has positive Lyapunov exponents for a.e. $x$ in $I$.

THEOREM 5.2. - The measure $\mu_{0}$ is ergodic and it is the unique $f$ invariant probability measure absolutely continuous with respect to Lebesgue.

Proof. - Since $\mu_{0}$ has positive Lyapunov exponents a.e. and satisfies (A3), we can use a theorem due to Ledrappier [7] in the form of part (3) of Proposition 3.3 in [16] to assert that $\mu_{0}$ is measure theoretically mixing and so it is ergodic.

We claim that $\mu_{0}$ is equivalent to the Lebesgue measure $m$ on $I_{*}=\left[c_{1}^{-}, c_{1}^{+}\right]$. This can be seen as follows; since $\varphi_{0}$ has Bounded Variation, and $\int \varphi_{0} \mathrm{~d} m_{0}=1$, there is some interval $\gamma \subset \widehat{W}$, such that $\inf _{\gamma}\left(\varphi_{0}\right)>0$ so the density of $\hat{\mu}_{0}$ with respect to the usual length is bounded away from zero on $\gamma$, as a consequence, $\inf _{\pi(\gamma)} \mathrm{d} \mu_{0} / \mathrm{d} m>0$. On the other hand, (A3) ensures that $f^{N}(\pi(\gamma))=I_{*}$ for some $N \geqslant 1$. Therefore

$$
\inf _{I_{*}} \frac{\mathrm{d} \mu_{0}}{\mathrm{~d} m} \geqslant \inf _{\pi(\gamma)} \frac{\mathrm{d} \mu_{0}}{\mathrm{~d} m} \frac{1}{\sup \left(f^{N}\right)^{\prime}}>0,
$$

which implies our claim.

Now, let $v$ be any $f$-invariant probability measure which is absolutely continuous with respect to Lebesgue measure. It is easy to see that the support of $v$ must be contained in $I_{*}$, and so $v \ll \mu_{0}\left(\mu_{0}\right.$ is equivalent to $m$ on $\left.I_{*}\right)$. It follows that $v=\mu_{0}$ because ergodic measures are minimal for the absolute continuity relation. That proves uniqueness.

Now, joining Theorem 5.1 and 5.2, Theorem A is proved.

Finishing this section we prove a property of the support of the function $\varphi_{0}$. Set

$$
\begin{aligned}
W_{\delta} & =\widehat{W} \backslash\left(\left(f(-\delta), c_{1}^{+}\right) \cup\left(c_{1}^{-}, f(\delta)\right)\right) \times\{0\} \\
& =\widehat{W} \backslash[f(\delta), f(-\delta)] \times\{0\} .
\end{aligned}
$$

LEMMA 5.2 (Vi 5.9). - The density $\varphi_{0}$ satisfies

(1) $\inf \left(\left.\varphi_{0}\right|_{[f(\delta), f(-\delta)] \times\{0\}}\right)>0$;

(2) $\inf \left(\left.\varphi_{0}\right|_{W_{k}^{ \pm}}\right)>0$, for every $k \geqslant 1$.

Proof. - Let $\gamma_{1} \subset \widehat{W}$ be an interval such that $\inf \left(\left.\varphi_{0}\right|_{\gamma_{1}}\right)>0$. By the topological mixing assumption (A3), there exists $n_{1} \geqslant 0$ such that 
$\pi\left(\hat{f}^{n_{1}}\left(\gamma_{1}\right)\right)=f^{n_{1}}\left(\pi\left(\gamma_{1}\right)\right)=I_{*}=\left[c_{1}^{-}, c_{1}^{+}\right]$. In particular, $\pi\left(\hat{f}^{n_{1}}\left(\gamma_{1}\right)\right)$ contains the fixed points of $f^{2}$, namely $p_{1}$ and $p_{2}$ with $p_{1}>p_{2}$. Moreover, up to slightly modifying $\beta$ if necessary, we may suppose that no endpoint of levels $E_{k}^{ \pm}$, for $k \geqslant 1$ projects down to $p_{1}$, nor $p_{2}$. Then there exists an open interval $\gamma_{2} \subset \hat{f}^{n_{1}}\left(\gamma_{1}\right)$ such that $\pi\left(\gamma_{2}\right)$ contains $p_{1}$. Clearly $\pi\left(\hat{f}^{2 n}\left(\gamma_{2}\right)\right)$ must contain $p_{1}$ for every $n \geqslant 0$. Now, suppose that $p_{i} \neq c_{k}^{ \pm}$for every $k \geqslant 1$ and for $i=1,2$. If this is not true, we simply replace $\left\{p_{i}: i=1,2\right\}$ by another periodic orbit not intersecting $(-\delta, \delta)$, and the argument proceeds along the same lines. Now we have that, there exists some finite time $n_{2} \geqslant 0$ such that $\hat{f}^{2 n_{2}}(\xi)=\left(p_{1}, 0\right)$, where $\xi \in \gamma_{2}$ satisfies $\pi(\xi)=p_{1}$. Up to another arbitrarily small modification of $\beta$, we may suppose that the orbit of $\xi$ does not pass trough any of the boundary points of the monotonicity intervals in $\mathcal{P}^{(1)}$. Therefore $\hat{f}^{2 n_{2}}\left(\pi\left(\gamma_{2}\right)\right)$ contains some open neighborhood $\gamma_{3}$ of $\left(p_{1}, 0\right)$ in $E_{0}$. Let $n_{3} \geqslant 0$ be the minimum time such that $\hat{f}^{2 n_{3}}\left(\pi\left(\gamma_{3}\right)\right)$ intersects $(-\delta, \delta)$. Hence

$$
\hat{f}^{2 n_{3}}\left(\gamma_{3}\right)=f^{2 n_{3}}\left(\pi\left(\gamma_{3}\right)\right) \times\{0\} \text { contains }\left[\delta, p_{1}\right] \times\{0\} .
$$

Set $\sigma_{1}=\hat{f}\left(\left[\delta, p_{1}\right] \times\{0\}\right)=\left[f(\delta), p_{2}\right] \times\{0\}$, so $\sigma_{1} \subset \hat{f}^{n+1}\left(\gamma_{1}\right)$ where $n=n_{1}+2 n_{2}+2 n_{3}$.

Now, with similar arguments we can set $\sigma_{2}=\hat{f}\left(\left[p_{2},-\delta\right] \times\{0\}\right)=$ $\left[p_{1}, f(-\delta)\right] \times\{0\}$, with the property that $\sigma_{2} \subset \hat{f}^{m+1}\left(\gamma_{1}\right)$ with $m=$ $n_{1}+2 m_{2}+2 m_{3}$, for some $m_{2}$ and $m_{3}$. Set also $\sigma_{3}=\left(p_{2}, p_{1}\right)$ and note that $\hat{f}\left(\sigma_{1} \cup \sigma_{2}\right)$ contains $\sigma_{3}$.

Now, since $\varphi_{0}$ is a fixed point for the transfer operator associated to $\hat{f}$, we have that $\inf \left(\left.\varphi_{0}\right|_{\gamma}\right)>0$ implies that $\inf \left(\left.\varphi_{0}\right|_{\hat{f}(\gamma)}\right)>0$, thus $\inf \left(\left.\varphi_{0}\right|_{\sigma_{i}}\right)>0$ for $i=1,2,3$, and part (1) follows immediately.

Moreover, given $(y, k) \in W_{k}^{ \pm}, k \geqslant 1$, and $z \in(-\delta, \delta)$ such that $f^{k}(z)=y$.

$$
\varphi_{0}(y, k)=\mathcal{L}_{0}^{k}\left(\varphi_{0}\right)(y, k)=\frac{\varphi_{0}(z, 0)}{\lambda^{k}} \geqslant \frac{1}{\lambda^{k}} \inf \left(\left.\varphi_{0}\right|_{[f(-\delta), f(\delta)] \times\{0\}}\right),
$$

which proves part (2).

This last relation also yields another useful conclusion, namely

$$
\varphi_{0}(y, k) \leqslant \frac{1}{\lambda^{k}} \sup \left(\left.\varphi_{0}\right|_{[f(-\delta), f(\delta)] \times\{0\}}\right) \leqslant \frac{1}{\lambda^{k}} \sup \left(\varphi_{0}\right)
$$


and so

$$
\sum_{k=1}^{\infty} \sup \left(\left.\varphi_{0}\right|_{E_{k}^{ \pm}}\right) \leqslant \sum_{k=1}^{\infty} \frac{1}{\lambda^{|k|}} \sup \left(\varphi_{0}\right),
$$

leading to

$$
\begin{aligned}
\sup \left(\varphi_{0}\right) & =\sum_{k=1}^{\infty} \sup \left(\left.\varphi_{0}\right|_{E_{k}^{+}}\right)+\sum_{k=1}^{\infty} \sup \left(\left.\varphi_{0}\right|_{E_{k}^{-}}\right)+\sup \left(\left.\varphi_{0}\right|_{E_{0}}\right) \\
& \leqslant \sum_{k=0}^{\infty} 2 \lambda^{-k} \sup \varphi_{0} .
\end{aligned}
$$

Note that Lemma 5.2 implies that $W_{\delta} \subset \operatorname{supp} \varphi_{0}$ and from this $\operatorname{supp} \varphi_{0}=W_{\delta}$, since $\varphi_{0}=\mathcal{L}^{n} \varphi_{0}$ implies that $\varphi_{0}$ is identically zero on $\hat{I} \backslash \hat{f}^{n}(\hat{I})$ for every $n \geqslant 1$ and discussion on p. 6 implies that $\bigcup_{n} \geqslant \hat{f}^{n}(\hat{I}) \subset$ $W_{\delta}$.

\section{DECAY OF CORRELATIONS}

In this section we prove that the measures $\hat{\mu}_{0}$ and $\mu_{0}$, that we have just constructed, are exact, and so, also mixing, for the corresponding dynamical systems $\hat{f}$ and $f$, respectively, in the same lines as stated in Proposition 5.13 of [14]. As a consequence, the transfer operator $\mathcal{L}_{0}$ is quasi-compact and both systems $\left(\hat{f}, \hat{\mu}_{0}\right)$ and $\left(f, \mu_{0}\right)$ have exponential decay of correlations in corresponding spaces of functions with bounded variation. This proposition also provides another proof of the ergodicity of $\mu_{0}$ (besides implying that $\hat{\mu}_{0}$ is also ergodic). We are not going to prove the equivalent of Proposition 5.13 of [14], because it follows the same arguments, provided that we prove some previous lemmas. Before proving these lemmas, let us make some conventions that will be used throughout this section. Set

$$
E_{k}= \begin{cases}E_{0} & \text { if } k=0 \\ E_{k}^{+} & \text {if } k \geqslant 1 \\ E_{|k|}^{-} & \text {if } k \leqslant-1\end{cases}
$$

and also denote by $\partial_{k}$ the set of boundary points of the elements of the partition $\mathcal{P}^{(1)}$. More precisely

$$
\partial_{0}=\{q,-\delta, 0, \delta,-q\}=\partial D_{0}^{L} \cup \partial U_{0}^{L} \cup \partial U_{0}^{R} \cup \partial D_{0}^{R},
$$


and for each $k \geqslant 1$

$$
\partial_{k}^{ \pm}=\partial D_{k}^{L \pm} \cup \partial U_{k}^{ \pm} \cup \partial D_{k}^{R \pm}
$$

Therefore $\partial_{k}$ can be expressed as follows

$$
\partial_{k}= \begin{cases}\partial_{0} & \text { if } k=0 \\ \partial_{k}^{+} & \text {if } k \geqslant 1 \\ \partial_{|k|}^{-} & \text {if } k \leqslant-1\end{cases}
$$

Observe that each $\partial_{k},|k| \geqslant 1$, contains at most eight points.

Now, for $n \geqslant 1, N \geqslant 1$ and $\eta \in \mathcal{P}^{(n+N)}$, let $(k(i))_{i}$ be the sequence given by

$$
\hat{f}^{i}(\eta) \subset E_{k(i)} \text { for each } i \geqslant 0 .
$$

Let $\tau>0$ be fixed and define $\mathcal{Q}(n, N)$ to be the subset of intervals $\eta \in \mathcal{P}^{(n+N)}$ such that:

(i) $|k(i)| \leqslant N+(n-i) \tau$ for $0 \leqslant i \leqslant n$.

(ii) $\hat{f}^{i}(\partial \eta)$ is disjoint from $\partial_{k(i)}$ for every $0 \leqslant i \leqslant n$.

LEMMA 6.1 (Vi 5.10). - Given $\varepsilon>0$ there exists $N \geqslant 0$, such that for each $n \geqslant 1$ the set $\mathcal{Q}(n, N)$ satisfies the following properties:

(1) for every $\eta \in \mathcal{Q}(n, N)$, we have $f^{n}(\eta) \in \mathcal{P}^{(N)} \subset \bigcup_{|k| \leqslant N} E_{k}$;

(2) the $\hat{\mu}_{0}$-measure of the union of the intervals $\eta \notin \mathcal{Q}(n, N)$ is at most $\varepsilon$.

Proof. - The statement of this lemma is not exactly the same as Lemma 5.10 of [14], but it is equivalent. The proof comes along the same arguments.

LEMMA 6.2 (Vi 5.11). - Given $n \geqslant 1$ and $\varepsilon_{2}>0$ there exists $\varepsilon_{1}>0$ for any $n \geqslant 1$, any interval $\eta \in \mathcal{Q}(n, N)$, and any borel subset $\xi \subset \eta$,

$$
\frac{m(\xi)}{m(\eta)} \leqslant \varepsilon_{1} \Rightarrow m\left(\hat{f}^{n}(\xi)\right) \leqslant \varepsilon_{2} .
$$

Proof. - Most of the proof is based on the same ideas as Lemmas 3.1 and 3.2. The main new ingredient is to use condition (i) $|k(i)| \leqslant n+$ $(n-i) \tau$ in the definition of $\mathcal{Q}(n, N)$, taking $\tau$ small enough, e.g., $\tau \leqslant \log \lambda \rho / \log 8$.

Suppose that $\eta \subset E_{0}$ and $\hat{f}^{n}(\eta) \subset E_{0}$. In this case we prove that $\hat{f}^{n}$ has uniformly bounded distortion on $\eta$ (depending on $N$, but not on $n$ 
or $\eta$ ). Let us consider the sequence of iterates $0 \leqslant v_{1}<v_{1}+p_{1}<v_{2}<$ $\cdots<v_{r}<v_{r}+p_{r}<n$ defined by

(a) $\hat{f}^{j}(\eta) \subset E_{0}$ for $0 \leqslant j \leqslant v_{1}$, for $v_{i}+p_{i}<j \leqslant v_{i+1}$ and $1 \leqslant i \leqslant$ $r-1$, and for $v_{r}+p_{r}<j \leqslant n$.

(b) $\hat{f}^{j}(\eta) \subset E_{j-v_{i}}$ if $\hat{f}^{v_{i}}(\eta) \subset(-\delta, 0)$ and $\hat{f}^{j}(\eta) \subset E_{-\left(j-v_{i}\right)}$ if $\hat{f}^{\nu_{i}}(\eta) \subset(0, \delta)$, for $v_{i} \leqslant j \leqslant v_{i}+p_{i}$, and $1 \leqslant i \leqslant r$,

Let $\gamma=\pi(\eta) \subset I$ and $x, y \in I$.

We first consider $0 \leqslant j<v_{1}$. Suppose $x<y$, since $f$ has negative Schwarzian derivative in $\gamma$ and from condition (A0), we have

$$
\begin{aligned}
& \sum_{j=0}^{v_{1}-1} \log f^{\prime}\left(f^{j}(x)\right)-\log f^{\prime}\left(f^{j}(y)\right) \\
& \quad=\sum_{j=0}^{v_{1}-1} \log \left(1+\frac{f^{\prime}\left(f^{j}(x)\right)-f^{\prime}\left(f^{j}(y)\right)}{f^{\prime}\left(f^{j}(y)\right)}\right) \\
& \leqslant \sum_{j=0}^{\nu_{1}-1} \frac{\left|f^{\prime}\left(f^{j}(x)\right)-f^{\prime}\left(f^{j}(y)\right)\right|}{f^{\prime}\left(f^{j}(y)\right)} \leqslant \sum_{j=0}^{\nu_{1}-1} A \frac{\left|x_{j}-y_{j}\right|}{K_{2}\left|y_{j}\right|}
\end{aligned}
$$

where $x_{j}=f^{j}(x)$, and $y_{j}=f^{j}(y)$.

But $y_{j} \notin(-\delta, \delta)$ gives $1 /\left|y_{j}\right|<1 / \delta$ and

$$
\left|f^{\nu_{1}-j}\left(f^{j}(x)\right)-f^{\nu_{1}-j}\left(f^{j}(y)\right)\right|=\left(f^{\nu_{1}-j}\right)^{\prime}(z)\left|x_{j}-y_{j}\right|
$$

for some $z \in f^{j}(\gamma)$, which implies $\left|f^{\nu_{1}}(\gamma)\right| \geqslant b \sigma_{0}^{\nu_{1}-j}\left|f^{j}(\gamma)\right|$ using Lemma 3.1, and leads to

$$
\begin{aligned}
\sum_{j=0}^{\nu_{1}-1} \log f^{\prime}\left(f^{j}(x)\right)-\log f^{\prime}\left(f^{j}(y)\right) & \leqslant \sum_{j=0}^{\nu_{1}-1} \operatorname{const} \sigma^{j-\nu_{1}}\left|f^{\nu_{1}}(\gamma)\right| \\
& \leqslant C\left|f^{\nu_{1}}(\gamma)\right| .
\end{aligned}
$$

Similar arguments show

$$
\sum_{j=0}^{\nu_{1}-1} \log f^{\prime}\left(f^{j}(y)\right)-\log f^{\prime}\left(f^{j}(x)\right) \leqslant C\left|f^{\nu_{1}}(\gamma)\right| .
$$

Thus

$$
\sum_{j=0}^{\nu_{1}-1}\left|\log f^{\prime}\left(f^{j}(x)\right)-\log f^{\prime}\left(f^{j}(y)\right)\right| \leqslant C\left|f^{\nu_{1}}(\gamma)\right| .
$$


And for the same reasons we have

$$
\sum_{j=v_{i}+p_{i}+1}^{v_{i+1}-1}\left|\log f^{\prime}\left(f^{j}(x)\right)-\log f^{\prime}\left(f^{j}(y)\right)\right| \leqslant C\left|f^{v_{i+1}}(\gamma)\right|
$$

for every $1 \leqslant i \leqslant r-1$, and also

$$
\sum_{j=v_{r}+p_{r}+1}^{n-1}\left|\log f^{\prime}\left(f^{j}(x)\right)-\log f^{\prime}\left(f^{j}(y)\right)\right| \leqslant C\left|f^{n}(\gamma)\right| .
$$

Now, let $j=v_{i}$ and denote $\Delta_{i}=d\left(f^{v_{i}}(\gamma), 0\right)$. Thus,

$$
\log f^{\prime}\left(f^{j}(x)\right)-\log f^{\prime}\left(f^{j}(y)\right) \leqslant \frac{f^{\nu_{i}}(\gamma)}{\Delta_{i}} .
$$

Next, we consider $v_{i}<j \leqslant v_{i}+p_{i}$. We are assuming that $f^{v_{i}}(\gamma) \subset$ $(-\delta, 0)$, therefore, in this case we have

$$
\sum_{j=v_{i}+1}^{v_{i}+p_{i}} \log f^{\prime}\left(f^{j}(x)\right)-\log f^{\prime}\left(f^{j}(y)\right) \leqslant \frac{A}{K_{2}} \sum_{j=v_{i}+1}^{v_{i}+p_{i}} \frac{\left|f^{j}(x)-f^{j}(y)\right|}{\left|f^{j}(y)\right|} .
$$

Let us see that the terms in the the sum are bounded by $f^{v_{i}}(\gamma) / \Delta_{i}$. In fact, we have

$$
\left|f^{j}(x)-f^{j}(y)\right|=\left(f^{j-v_{i}}\right)^{\prime}\left(f^{v_{i}}(z)\right)\left|f^{v_{i}}(x)-f^{v_{i}}(y)\right|
$$

for some $z \in \gamma$, more precisely $z \in[x, y]$, as a consequence of the Mean Value Theorem. Now, the Chain Rule and condition (A0) imply

$$
\begin{aligned}
\left|f^{j}(x)-f^{j}(y)\right| & \leqslant\left(f^{j-v_{i}-1}\right)^{\prime}\left(f\left(f^{v_{i}}(z)\right)\right) \cdot f^{\prime}\left(f^{v_{i}}(z)\right)\left|f^{v_{i}}(\gamma)\right| \\
& \leqslant C\left(f^{j-v_{i}-1}\right)^{\prime}\left(c_{1}^{+}\right) \cdot K_{1}\left|f^{v_{i}}(z)\right|^{s-1}\left|f^{v_{i}}(\gamma)\right| .
\end{aligned}
$$

On the other hand, for this $z$ we have

$$
\begin{aligned}
\left|f^{j}(y)-c_{j-v_{i}}^{+}\right| & =\left|f^{j-1-v_{i}}\left(f^{v_{i}+1}(y)\right)-f^{j-1-v_{i}}\left(c_{1}^{+}\right)\right| \\
& =\left(f^{j-1-v_{i}}\right)^{\prime}(\tilde{z})\left|f\left(f^{v_{i}}(y)\right)-c_{1}^{+}\right| \\
& \geqslant \frac{1}{C}\left(f^{j-1-v_{i}}\right)^{\prime}\left(c_{1}^{+}\right) K_{2} \frac{\left|f^{v_{i}}(z)\right|^{s}}{s} .
\end{aligned}
$$

Thus we can write

$$
\left|f^{j}(x)-f^{j}(y)\right| \leqslant s C^{2} \frac{K_{1}}{K_{2}} \frac{\left|f^{j}(z)-c_{j-v_{i}}^{+}\right|}{\left|f^{\nu_{i}}(z)\right|^{s}}\left|f^{\nu_{i}}(z)\right|^{s-1}\left|f^{v_{i}}(\gamma)\right| .
$$


Now, since $v_{i}<j \leqslant v_{i}+p_{i}$, and $z \in[x, y] \subset \gamma$

$$
\left|f^{j}(x)-f^{j}(y)\right| \leqslant s C^{2} \frac{K_{1}}{K_{2}} \frac{\mathrm{e}^{-\beta\left(j-v_{i}\right)}}{\left|f^{v_{i}}(z)\right|}\left|f^{v_{i}}(\gamma)\right| .
$$

Therefore

$$
\frac{\left|f^{j}(x)-f^{j}(y)\right|}{\left|f^{j}(y)\right|} \leqslant C \frac{\mathrm{e}^{-\beta\left(j-v_{i}\right)}}{\left(\mathrm{e}^{-\alpha\left(j-v_{i}\right)}-\mathrm{e}^{-\beta\left(j-v_{i}\right)}\right)} \frac{\left|f^{\nu_{i}}(\gamma)\right|}{\Delta_{i}},
$$

since $f^{j}(y) \in E_{j-v_{i}}$.

That is

$$
\sum_{j=v_{i}+1}^{v_{i}+p_{i}} \frac{\left|f^{j}(x)-f^{j}(y)\right|}{\left|f^{j}(y)\right|} \leqslant C \frac{\left|f^{v_{i}}(\gamma)\right|}{\Delta_{i}}
$$

since $\beta>\alpha$.

Interchanging the roles of $x$ and $y$ in the above arguments, we have

$$
\sum_{j=v_{i}+1}^{v_{i}+p_{i}}\left|\log f^{\prime}\left(f^{j}(x)\right)-\log f^{\prime}\left(f^{j}(y)\right)\right| \leqslant \operatorname{const} \frac{\left|f^{v_{i}}(\gamma)\right|}{\Delta_{i}} .
$$

Thus, joining all the parts, we obtain

$$
\begin{aligned}
& \sum_{j=0}^{n-1}\left|\log f^{\prime}\left(f^{j}(x)\right)-\log f^{\prime}\left(f^{j}(y)\right)\right| \\
& \quad \leqslant \operatorname{const} \sum_{i=1}^{r} \frac{\left|f^{v_{i}}(\gamma)\right|}{\Delta_{i}}+\text { const } f^{n}(\gamma)
\end{aligned}
$$

of course $f^{n}(\gamma) \leqslant$ const, thus

$$
\left|f^{\nu_{i}}(\gamma)\right| \leqslant \text { const }(\lambda \rho)^{\nu_{i}-n}\left|f^{n}(\gamma)\right| \leqslant \operatorname{const}(\lambda \rho)^{\nu_{i}-n}
$$

for each $1 \leqslant i \leqslant r-1$, and from Lemmas 3.1 and 3.2.2.

Now, since $f^{p_{i}}\left(f^{v_{i}}(\gamma)\right) \subset D_{p_{i}}^{R-}$ we have

$$
\left|f^{p_{i}+1}\left(\Delta_{i}\right)-c_{p_{i}}^{+}\right| \geqslant \mathrm{e}^{-\beta\left(p_{i}+1\right)},
$$

which implies (assuming that $f^{\prime} \leqslant 4$ )

$$
4^{p_{i}} \Delta_{i}^{s} \frac{K_{1}}{s} \geqslant\left(f^{p_{i}}\right)^{\prime}(\tilde{z})\left|f\left(\Delta_{i}\right)-c_{1}^{+}\right| \geqslant \mathrm{e}^{-\beta\left(p_{i}+1\right)} .
$$


This last relation leads to

$$
\Delta_{i}^{s} \geqslant \frac{K_{1}}{s} \mathrm{e}^{-\beta\left(p_{i}+1\right)} 4^{-p_{i}} \geqslant \text { const } \mathrm{e}^{-2 \beta p_{i}} 4^{-p_{i}} .
$$

Now, condition (i) in the definition of $\mathcal{Q}(n, N)$ implies $p_{i}=|k(i)| \leqslant$ $N+\left(n-v_{i}-p_{i}\right) \tau \leqslant N+\left(n-v_{i}\right)$ and since we have chosen $\tau=$ $\log \lambda \rho / \log 8$ and $\mathrm{e}^{\beta}<\lambda_{c}^{1 / s} \leqslant 2$, we obtain

$$
\begin{aligned}
\sum_{i=1}^{r} \frac{\left|f^{v_{i}}(\gamma)\right|}{\Delta_{i}} & \leqslant \sum_{i=1}^{r}(\lambda \rho)^{v_{i}-n}\left(2 \mathrm{e}^{\beta}\right)^{p_{i}} \leqslant \sum_{i=1}^{r} 4^{N}\left(\lambda \rho 4^{-\tau}\right)^{v_{i}-n} \\
& \leqslant 4^{N} \sum_{i=1}^{r} 2^{\left(v_{i}-n\right) \tau} \leqslant \mathrm{const} 4^{N} .
\end{aligned}
$$

Replacing in (9) we conclude that $f^{n}$ has bounded distortion on $\gamma$

$$
\sum_{j=0}^{n-1}\left|\log f^{\prime}\left(f^{j}(x)\right)-\log f^{\prime}\left(f^{j}(y)\right)\right| \leqslant \text { const } 4^{N} .
$$

In equivalent terms, $\hat{f}^{n}$ has bounded distortion on $\eta$ as we had claimed. In particular, in this case we may take $\varepsilon_{1}=\left(\varepsilon_{2} / m(I)\right) \exp \left(-\tilde{K}_{1}\right)$, where $\tilde{K}_{1}>0$ denotes the right hand term in (10).

Now, the remaining cases can be treated easily. If $\eta$ is not contained in $E_{0}$ then we define $\left(p_{0}+1\right) \geqslant 1$ to be the first iterate for which $\hat{f}^{p_{0}+1}(\eta) \subset E_{0}$. Then, we modify the first condition in (a) to $\hat{f}^{j}(\eta) \subset E_{0}$ for $p_{0}+1 \leqslant j \leqslant v_{1}$. Therefore, the sum

$$
\sum_{j=p_{0}+1}^{\nu_{1}-1}\left|\log f^{\prime}\left(f^{j}(x)\right)-\log f^{\prime}\left(f^{j}(y)\right)\right|
$$

can be estimated in just the same way as (6).

For the sum over $0 \leqslant j \leqslant p_{0}$ it is used a simpler version of (8), since $\hat{f}^{j}(\eta) \subset E_{k(0)+j}$, if $k(0)>0$, and $E_{k(0)-j}$, if $k(0)<0$, and for $k(0)=0$ we have to choose between $E_{j}$ or $E_{-j}$ depending upon $f^{j}(\eta)$ is to the left or to the right side of the critical point. From this,

$$
\begin{aligned}
& \sum_{j=0}^{p_{0}} \log f^{\prime}\left(f^{j}(x)\right)-\log f^{\prime}\left(f^{j}(y)\right) \\
& \quad \leqslant \frac{A}{K_{2}} \sum_{j=0}^{p_{0}} \frac{\left|f^{j}(x)-f^{j}(y)\right|}{\left|f^{j}(y)\right|} \leqslant \frac{A}{K_{2}} \text { const } \sum_{j=0}^{p_{0}} \mathrm{e}^{-(\beta-\alpha)(j+|k(0)|)}
\end{aligned}
$$




$$
\leqslant \sum_{j=0}^{p_{0}} \mathrm{e}^{-(\beta-\alpha)(j+|k(0)|)} \leqslant \text { const. }
$$

Thus, this last sum just adds a constant term to (9), and so does not affect the conclusion (10): $\hat{f}^{n}$ has bounded distortion on $\eta$ also in this case.

Finally, suppose that $\hat{f}^{n}(\eta)$ is not contained in $E_{0}$. Then let $v=v_{r}$ be the last iterate for which $\hat{f}^{v}(\eta) \subset E_{0}$, and we do not define $p_{r}$. The previous cases show us that $\hat{f}^{v}$ has bounded distortion on $\eta$, see (10)

$$
\sum_{j=0}^{\nu-1}\left|\log f^{\prime}\left(f^{j}(x)\right)-\log f^{\prime}\left(f^{j}(y)\right)\right| \leqslant \tilde{K}_{1} .
$$

From this point on, we can follow the arguments in [14] to conclude the proof of the lemma.

Let $\mathcal{B}$ the Borel $\sigma$-algebra of $I$ and $\hat{\mathcal{B}}$ the Borel $\sigma$-algebra of $\hat{I}$. By definition, the invariant measure $\mu_{0}$ is exact for $f$ if

$$
B \in \mathcal{B}_{\infty}=\bigcap_{n=0}^{\infty} f^{-n}(\mathcal{B}) \Rightarrow \mu_{0}(B)=0 \quad \text { or } \mu_{0}(I \backslash B)=0 .
$$

Analogously, we say that $\hat{\mu}_{0}$ is exact for $\hat{f}$ if

$$
\hat{B} \in \hat{\mathcal{B}}_{\infty}=\bigcap_{n=0}^{\infty} f^{-n}(\hat{\mathcal{B}}) \Rightarrow \hat{\mu}_{0}(\hat{B})=0 \quad \text { or } \hat{\mu}_{0}(I \backslash \hat{B})=0 .
$$

LEMMA 6.3 (Lemma 5.12). -

(1) If $A \subset I$ belongs to $\mathcal{B}$ then $\pi^{-1}(A) \subset \hat{I}$ belongs to $\hat{\mathcal{B}}_{\infty}$.

(2) For any $\hat{A} \subset \hat{I}$ in $\hat{\mathcal{B}}_{\infty}$ there are $A_{1} \subset A_{2} \subset I$ so that $\pi^{-1}\left(A_{1}\right) \subset$ $\hat{A} \subset \pi^{-1}\left(A_{2}\right)$ and $A_{2} \backslash A_{1}$ is a countable set.

Proof. - The first part is easy. In fact if $A=f^{-n}\left(A_{n}\right)$ for some Borel subset $A_{n} \subset I$ then $x \in \pi^{-1}\left(A_{n}\right)$ if and only if $\pi(x) \in A$ if and only if $\pi\left(\hat{f}^{n}(x)\right)=f^{n}(\pi(x)) \in A_{n}$ if and only if $\hat{f}^{n}(x) \in \pi^{-1}\left(A_{n}\right)$ which is equivalent to $x \in \hat{f}^{-n}\left(\pi^{-1}\left(A_{n}\right)\right)$.

That is $\pi^{-1}(A)=\hat{f}^{-n}\left(\pi^{-1}\left(A_{n}\right)\right)$.

To proof part (2), let $A_{2}=\pi(\hat{A})$ and

$$
A_{1}=A_{2} \backslash \bigcup_{n=0}^{\infty} f^{-n}\left\{0, c_{j}^{+}, c_{j}^{-}: j \geqslant 0\right\} .
$$


It is clear that $\hat{A} \subset \pi^{-1}\left(A_{2}\right)$, so let us prove that $\pi^{-1}\left(A_{1}\right) \subset \hat{A}$.

Given any $z \in A_{1}$ there is some $\xi \in \hat{A}$ such that $\pi(\xi)=z$. Thus we only have to show that any other $\eta \in \hat{I}$ such that $\pi(\eta)=z$ also belongs in $\hat{A}$. Now, the elements of $\hat{\mathcal{B}}_{\infty}$ are characterized by the property

$$
\left[\zeta_{1} \in \hat{A} \text { and } \hat{f}^{n}\left(\zeta_{1}\right)=\hat{f}^{n}\left(\zeta_{2}\right) \text { for some } n \geqslant 1\right] \Rightarrow \zeta_{2} \in \hat{A} \text {. }
$$

Therefore, we are left to show that for any $\xi$ and $\eta$ as above, there is $n \geqslant 1$ such that $\hat{f}^{n}(\xi)=\hat{f}^{n}(\eta)$. To this end, since $\pi\left(\hat{f}^{n}(\xi)\right)=\pi\left(\hat{f}^{n}(\eta)\right)$ for every $n \geqslant 1$, it suffices to show that there exists $n \geqslant 1$ such that $\hat{f}^{n}(\xi)$ and $\hat{f}^{n}(\eta)$ are both in $E_{0}$.

To proof the above assertion we introduce the following notion: Given $x \in(-\delta, \delta)$, we define the falling time $p(x)$ of $x$ to be the smallest integer $j \geqslant 1$ such that $\hat{f}^{j+1}(x, 0) \in E_{0}$. The same kind of argument as in (3) gives, recall A1,

$$
\begin{aligned}
\mathrm{e}^{-\beta p(x)} & \geqslant\left|f^{p(x)}(x)-c_{p(x)}^{ \pm}\right| \geqslant \frac{1}{C}\left(f^{p(x)-1}\right)^{\prime}\left(c_{1}^{ \pm}\right)\left|c_{1}^{ \pm}-f(x)\right| \\
& \geqslant \frac{1}{C \lambda_{c}} \lambda_{c}^{p(x)} K_{2} \frac{|x|^{s}}{s} .
\end{aligned}
$$

Set $\gamma=1-\mathrm{e}^{\alpha-\beta}>0$. Up to taking $\delta$ small, we may suppose that $p(x) \geqslant H(\delta)$ is large enough so that the previous relation implies

$$
\lambda_{c}^{p(x)}|x|^{s} \leqslant \gamma^{s} .
$$

in particular $x \neq 0$ implies $p(x)<\infty$.

Now, write $\xi=(z, k)$ and $\eta=(z, l)$. The definition of $A_{1}$ ensures that the $f$-orbit of $z \in A_{1}$ is disjoint from the critical orbit, and so $p\left(f^{n}(z)\right)$ is finite for every $n \geqslant 1$. Suppose that there is no $n \geqslant 1$ such that both $\hat{f}^{n}(\xi)$ and $\hat{f}^{n}(\eta)$ are in $E_{0}$. Then each of their orbits must start climbing the tower (in its corresponding block), before the other falls down to $E_{0}$. That is, there must be an infinite sequence (in order not to have $\hat{f}^{n}(\xi)$ and $\hat{f}^{n}(\eta)$ both in $E_{0}$ ) of times $0<v_{1}<v_{2}<\cdots$ such that $f^{v_{i}}(z) \in(-\delta, \delta)$ (one of the orbits moves from $E_{0}$ to $E_{1}$ or to $E_{-1}$ ) and $v_{i+1} \leqslant v_{i}+p\left(f^{v_{i}}(z)\right)$ (while the other is still climbing up) for all $i \geqslant 1$.

To check that this leads to a contradiction, we write $p_{i}=p\left(f^{v_{i}}(z)\right)$ and note that if $v_{i+1}-v_{i} \leqslant p_{i}$ then $\left|f^{v_{i+1}}(z)-c_{\left(v_{i+1}-v_{i}\right)}\right| \leqslant \mathrm{e}^{-\beta\left(v_{i+1}-v_{i}\right)}$. So we have

$$
\begin{aligned}
\left|f^{v_{i+1}}(z)\right| & \geqslant \mathrm{e}^{-\alpha\left(v_{i+1}-v_{i}\right)}-\mathrm{e}^{-\beta\left(v_{i+1}-v_{i}\right)} \\
& \geqslant \mathrm{e}^{-\alpha\left(v_{i+1}-v_{i}\right)}\left(1-\mathrm{e}^{-(\beta-\alpha)\left(v_{i+1}-v_{i}\right)}\right)
\end{aligned}
$$




$$
\geqslant \mathrm{e}^{-\alpha\left(v_{i+1}-v_{i}\right)} \gamma \geqslant \gamma \mathrm{e}^{-\alpha p_{i}}
$$

and in the last implication we use (A2).

Combining this with (13) and $\mathrm{e}^{s \alpha /(s-1)}<\lambda_{c}^{1 / s}$, we get

$$
\gamma^{s} \geqslant \lambda_{c}^{p_{i+1}}\left|f^{v_{i+1}}(z)\right|^{s} \geqslant \gamma^{s} \lambda_{c}^{p_{i+1}}\left(\mathrm{e}^{-s \alpha}\right)^{p_{i}} \geqslant \gamma^{s} \lambda_{c}^{p_{i+1}} \lambda_{c}^{-(s-1) p_{i} / s} .
$$

The last term is greater than or equal to $\lambda^{p_{i+1}-p_{i}(s-1) / s}$ which implies $p_{i+1} \leqslant p_{i}(s-1) / s$ for every $i \geqslant 1$. Since $p_{i}$ are positive integers, the sequence $\left(p_{i}\right)_{i}$ can not be infinite. This gives the contradiction we are looking for.

Now, Propositions 5.13 (exactness), 5.14 (quasi-compacity), 5.15 (decay of correlations) of [14] and also the Central Limit Theorem are deduced with the same arguments.

\section{THE SRB MEASURE FOR THE CONTRACTING LORENZ ATTRACTOR}

Nowadays there exists many literature about the strange attractor first discovered by Lorenz [8], as a truncation of a Navier-Stokes equation. One of them is the geometric model introduced by Guckenheimer and Williams in [3], called the Expanding Lorenz Attractor. More explicitly, they found a family of $C^{r}\left(\mathbb{R}^{3}\right)$ vector fields such that it is linear in a neighborhood of the origin containing the cube $\{(x, y, z) \in$ $\left.\mathbb{R}^{3}:|x|,|y|,|z| \leqslant 1\right\}$ and with eigenvalues $\lambda_{1}, \lambda_{2}, \lambda_{3}$ satisfying $\lambda_{2}<$ $\lambda_{1}<0<\lambda_{3}$ and $\lambda_{1}+\lambda_{3}>0$, and with both trajectories of the unstable manifold intersecting the top of the cube, as in Fig. 1. So if we call $U$ the union of the cube with a neighborhood of the unstable manifold, there exists an attractor $\Lambda=\bigcap_{t \geqslant 0} X_{t}(U)$ where $X_{t}$ is the flow of the vector field.

The Contracting Lorenz Attractor arises in a similar way if we replace the expanding condition $\lambda_{1}+\lambda_{3}>0$ by the contracting condition $\lambda_{1}+$ $\lambda_{3}<0$, see [11]. By construction, the top of the cube is a cross section $Q$ for the flow. More explicitly, there exist a curve $\Sigma$ (that we can assume to be the intersection $Q$ with the plane $\{x=0\})$. So there exist a first return map (a Poincare map) of the form

$$
\begin{aligned}
P: Q \backslash \Sigma & \rightarrow Q, \\
(x, y) & \mapsto P(x, y)=(f(x), g(x, y)),
\end{aligned}
$$




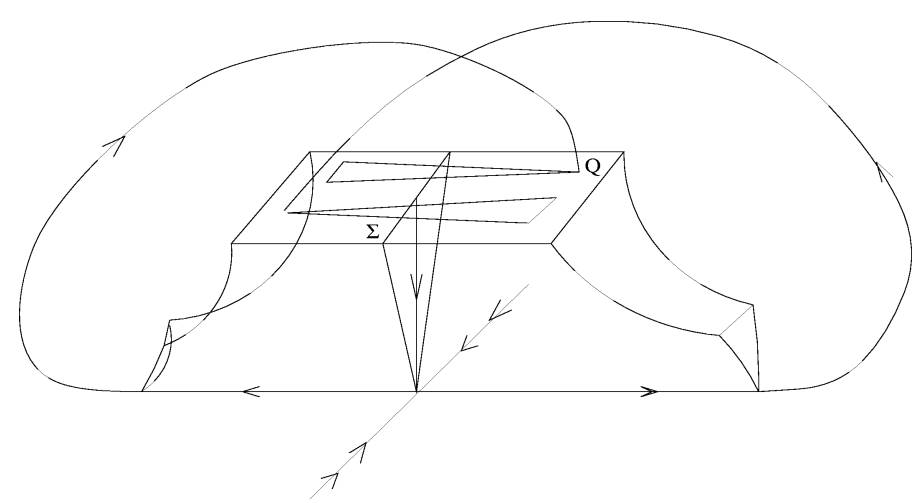

Fig. 1.

This Poincare map reduces in a wide sense the study of the dynamics of the Lorenz attractor to the study of the map $P$. But also the form of this map, that says that the leaves with $x=c t e$ are mapped to leaves with $x=f($ cte $)$, allows another simplification if we project along the stable leaves, see [11]. So, we can study the one-dimensional map defined by $f$.

By an SRB measure for the flow we mean a measure $v$, invariant by the flow, define on $\mathbb{R}^{3}$ such that its support is contained in the attractor and satisfying

$$
\lim _{T \rightarrow \infty} \frac{1}{T} \int_{0}^{T} \varphi\left(X_{t}(x)\right) \mathrm{d} t=\int \varphi \mathrm{d} \nu,
$$

for almost all $x$ contained in the basin of attraction $U$, and for every continuous function $\varphi: \mathbb{R}^{3} \rightarrow \mathbb{R}$.

To construct an SRB measure for this kind of flows we will assume that they define one-dimensional maps satisfying conditions (A0)-(A3). A. Rovella showed that this kind of flows have a kind of persistence, see [11]. So we are dealing with a wide class of flows.

So let $f$ be the projection along stable leaves of the first return map of the contracting Lorenz attractor. By Theorem A, $f$ has a SRB measure $\mu$. We can consider this measure as defined on the $\sigma$-algebra generated by sets containing whole stables leaves. If we consider the push forward of this measure by the Poincare map $P$, i.e., $P_{*} \mu(B)=\mu(B)$ we can take the weak ${ }^{*}$ limit of the sequence of measures $P_{*}^{n} \mu$ as a measure $\mu_{P}$ on the intersection of the attractor with the cross section $Q$, which is SRB. 
Now, we can saturate this measure along the flow in the following way. Let $\tau(z)$ be the return time of the point $z \in Q \backslash \Sigma$ so that $P(z)=X_{\tau(z)}(z)$. With this definition we take our measure $v$ in $U$ as

$$
\nu(B)=\frac{\int\left(\int_{0}^{\tau(z)} \chi_{B}\left(X_{t}(z)\right) \mathrm{d} t\right) \mathrm{d} \mu_{P}(z)}{\int \tau(z) \mathrm{d} \mu_{P}(z)} .
$$

The denominator is the term of normalization of the measure. This procedure gives a well define measure since $\mu$ is absolutely continuous with respect to the natural Lebesgue measure of the unstable manifold and of bounded density. The term of normalization is finite since $\tau(z) \approx \log (d(z, \Sigma))$. This is a standard procedure, see for example [14] Chapter 6.

With this construction it is not difficult to verify that $v$ is a SRB measure for the Contracting Lorenz Attractor. On the other hand, this measure is unique. In fact, if $v^{\prime}$ is another SRB measure we can define

$$
\mu^{\prime}=\left.\frac{\mathrm{d}}{\mathrm{d} t} v^{\prime}\left(\bigcup_{0 \leqslant s \leqslant t} X_{t}(\Gamma)\right)\right|_{t=0}
$$

for every borelian $\Gamma \subset Q$ and we will obtain an SRB measure on the section $Q$. Since this measure is unique we have $\mu^{\prime}=\mu$ and recovering the measure by means of the definition in (14) we also have $v^{\prime}=v$.

\section{ACKNOWLEDGEMENTS}

The author is grateful to Professor Jacob Palis and Professor Marcelo Viana for their support and many discussions.

\section{REFERENCES}

[1] Bowen R., Ruelle D., The ergodic theory of Axiom A flows, Inv. Math. 29 (1975) 181-202.

[2] Baladi V., Viana M., Strong stochastic stability and rate of mixing for unimodal maps, Ann. Scient. E.N.S. 29 (4) (1996) 483-517.

[3] Guckenheimer J., Williams R.F., Structural stability of Lorenz attractors, Publ. Math. IHES 50 (1979) 307-320.

[4] Katok A., Kifer Y., Random perturbations of transformations of an interval, J. de Analyse Math. 47 (1986) 193-237.

[5] Kifer Y., Ergodic Theory of Random Perturbations, Birkhäuser, Boston, 1986.

[6] Kifer Y., Random Perturbations of Dynamical Systems, Birkhäuser, Boston, 1988. 
[7] Ledrappier F., Some properties of absolutely continuos invariant measures on interval, Ergodic Theory Dynamical Systems 1 (1981) 77-93.

[8] Lorenz E.N., Deterministic non periodic flow, J. Atmosph. Sci. 20 (1963) 130-141.

[9] Palis J., A global view of dynamics and a conjecture on the denseness of finitude of attractors, Asterisque (1998).

[10] Robinson C., Transitivity and invariant measures for the geometric model of the Lorenz attractor, Ergodic Theory Dynamical Systems 4 (1984) 605-611.

[11] Rovella A., The dynamics of perturbations of the contracting Lorenz attractor, Bull. Brazil. Math. Soc. 24 (1993) 233-259.

[12] Ruelle D., A measure associated with Axiom A attractors, Amer. J. Math. 98 (1976) 619-654.

[13] Sinai Ya., Gibbs measure in ergodic theory, Russian Math. Surveys 27 (1972) 2179.

[14] Viana M., Stochastic Dynamics of Deterministic Systems, $21^{\circ}$ Colóquio Brasileiro de Matemática, IMPA, 1997.

[15] Viana M., Dynamics: a probabilistic and geometric perspective, Doc. Math. J. (Extra Volume ICM) (1998).

[16] Young L.-S., Decay of correlations for certain quadratic maps, Comm. Math. Phys. 146 (1992) 123-138. 